RESEARCH HIGHLIGHTS

HIV

\section{Complex regulation of immune responses to HIV and SIV}

The function of regulatory $\mathrm{T}$ cells in infectious diseases is a hot topic at present. In retroviral infection, it is not clear whether regulatory $\mathrm{T}$ cells suppress appropriate immune responses, in which case the elimination of these cells might be beneficial, or whether they can have a protective role, in which case impaired generation of regulatory $\mathrm{T}$ cells would contribute to the aberrant $\mathrm{T}$-cell activation that is a hallmark of HIV infection. Two recent studies emphasize the complexity of this issue in retroviral infections.

Reporting in The Journal of Immunology, Andersson and colleagues show that chronic infection with HIV can influence the tissue distribution of endogenous regulatory $\mathrm{T}\left(\mathrm{T}_{\text {Reg }}\right)$ cells. The authors analysed the role of $\mathrm{T}_{\mathrm{Reg}}$ cells in HIV infection by quantifying the lymphoid-tissue expression of three $\mathrm{T}_{\mathrm{Reg}}$-cell markers: CTLA4 (cytotoxic T-lymphocyte antigen 4), GITR (glucocorticoidinduced tumour-necrosis factor (TNF)-receptor-related protein) and the transcription factor FOXP3 (forkhead box P3). They found that, compared with patients who were treated with highly active antiretroviral therapy (HAART), patients in whom HIV was actively replicating had higher levels of these three $\mathrm{T}_{\text {Reg }}$-cell markers in their lymphoid tissues. The authors suggest that $\mathrm{T}_{\text {Reg }}$ cells are recruited to lymphoid organs in which HIV is actively replicating and that these $\mathrm{T}_{\mathrm{Reg}}$ cells could hamper the development of appropriate anti-HIV responses at these sites. This model indicates that elimination of $\mathrm{T}_{\mathrm{Reg}}$ cells should be beneficial for the development of protective immune responses.

In another study, which was reported in The Journal of Clinical Investigation, Kornfeld and colleagues assessed the correlates of protection against simian immunodeficiency virus (SIV) infection of African green monkeys (using the isolate $\left.\mathrm{SIV}_{\mathrm{AGM}}\right)$. This SIV infection of a natural African host is non-pathogenic; only SIV infections of African primate species result in non-pathogenic infections, which is in contrast to infection of Asian macaques with SIV $_{\text {MAC }}$, even though the viral loads are similar in these systems. In this study, the authors focused on assessing the early stages of SIV $_{\mathrm{AGM}}$ infection in African green monkeys. They examined patterns of T-cell activation and cytokine profiles in various tissues during primary $\mathrm{SIV}_{\mathrm{AGM}}$ infection. A transient increase in T-cell activation was detected but returned to a baseline level at $\sim 3$ weeks after infection. In contrast to infection with HIV, there were no significant increases in the levels of the chemokines CC-chemokine ligand 3 (CCL3) and CCL 4 or of the cytokine TNF. An early and strong induction of expression of transforming growth factor- $\beta$ and FOXP3 was detected, and this correlated with an increase in the numbers of $\mathrm{CD} 4^{+} \mathrm{CD} 25^{+}$and $\mathrm{CD} 8^{+}$ $\mathrm{CD} 25^{+} \mathrm{T}$ cells. Although functional studies are required to confirm the regulatory nature of these cells, the authors suggest that these results favour a protective role for regulatory T cells in controlling abnormal T-cell activation, which then leads to nonpathogenic infection. On the basis of this model, it might be possible to develop novel intervention strategies for the treatment of HIV infection.

Taken together, these studies, which investigated two different phases of retroviral infection, show the difficulties in identifying the correlates of protection against retroviral infection and in determining the role of regulatory $\mathrm{T}$ cells. It will be crucial to gain a better understanding of regulatory $\mathrm{T}$-cell function at different stages of infection for the design of appropriate therapeutics.

\section{Elaine Bell}

(2) References and links ORIGINAL RESEARCH PAPERS Andersson, J. et al. The prevalence of regulatory $T$ cells in lymphoid tissue is correlated with viral load in HIVinfected patients. J. Immunol. 174, 3143-3147 (2005) | Kornfeld, C. et al. Antiinflammatory profiles during primary SIV infection in African green monkeys are associated with protection against AIDS. J. Clin. Invest. 115, 1082-1091 (2005)

\section{IN BRIEF}

\author{
TUMOUR IMMUNITY
}

DC-NK cell cross-talk as a novel CD4+ ${ }^{+}$T cellindependent pathway for antitumour CTL induction.

Adam, C. et al. Blood 15 Mar 2005 (doi:10.1182/blood-2004-09-3775)

While assessing the ability of peptide-pulsed dendritic cells (DCs) to protect against tumour-cell challenge, Adam et al. observed that non-pulsed, syngeneic, bone-marrow-derived DCs protected mice against challenge with A20 B-cell lymphoma cells. Protection was natural killer (NK)-cell dependent, and surprisingly, protected mice showed A20-specific CD8 ${ }^{+}$T-cell-dependent immunological memory. Such memory was also observed after immunization with CD40-deficient DCs, indicating that $\mathrm{CD} 8^{+} \mathrm{T}$-cell activation occurred independently of CD4 ${ }^{+}$T-cell help. Because NK cells secreted interferon- $\gamma($ IFN- $\gamma$ ) in response to CD40-deficient DCs and because this was required for $\mathrm{CD} 8^{+} \mathrm{T}$-cell activation, the authors suggest that immunization with DCs can activate NK cells to produce IFN- $\gamma$, which then activates endogenous DCs to activate $\mathrm{CD} 8^{+} \mathrm{T}$ cells independently of $\mathrm{CD} 4^{+} \mathrm{T}$-cell help.

\section{INFLAMMATION}

The N-terminal domain of thrombomodulin sequesters high-mobility group-B1 protein, a novel antiinflammatory mechanism.

Abeyama, K. et al. J. Clin. Invest. 14 Apr 2005 (doi:1172/JCl200522782)

Abeyama et al. set out to investigate whether thrombomodulin (TM) - an endothelial-cell-specific membrane receptor that is an anticoagulant co-factor - also inhibits inflammation by sequestering pro-inflammatory mediators such as high-mobility group box 1 protein (HMGB1). They found that TM associates with HMGB1 through its amino (N)-terminal lectin-like domain (D1), which prevents HMGB1 from binding its receptor RAGE (receptor for advanced glycation end-products). Functional evidence to support their hypothesis was provided by the observation that both recombinant soluble human TM and a recombinant polypeptide corresponding to D1 inhibited HMGB1-induced activation of nuclear factor- $\mathrm{\kappa B}$ and generation of reactive-oxygen species in vitro, as well as ultraviolet-light-induced cutaneous inflammation and lipopolysaccharide-induced lethality in vivo.

\section{T-CELL SIGNALLING}

A ligand-induced conformational change in the $T$ cell receptor associated with productive immune synapses.

Risueño, R. M. et al. Blood 24 Mar 2005 (doi:10.1182/blood-2004-12-4763)

There are two main models for the initiation of T-cell receptor (TCR) signalling: the clustering model, in which TCR signalling is initiated by TCR clustering, a model that is supported by the ability of antibodies to crosslink the TCR and stimulate signalling; and the conformational-change model. So far, structural studies have not provided much evidence to support a role for conformational change. In this study, Risueño et al. show that the antibody APA1/1 detects a conformational change in the cytoplasmic tail of the CD3 $\varepsilon$ chain following TCR ligation. This change was only detectable when the TCR was stimulated with an agonist peptide in the context of MHC and not with a partial agonist peptide, and the change was detectable both in vitro and in vivo. 